\title{
Innovations in Cervical and Endometrial Cancer
}

\author{
Neuerungen beim Zervix- und Endometriumkarzinom
}

Authors

Affiliations
P. Mallmann ${ }^{1}$, M. W. Beckmann ${ }^{2}$, G. Emons ${ }^{3}$, für die Organkommission Uterus

1 Klinik und Poliklinik für Frauenheilkunde, Universitätsklinikum Köln, Köln

2 Frauenklinik Universitätsklinikum Erlangen, Erlangen

${ }^{3}$ Klinik für Gynäkologie und Geburtshilfe, Georg-August-Universität Göttingen, Göttingen

\section{Key words}

- surgical, drug and palliative therapy for cervical cancer

- surgical, adjuvant and palliative therapy for endometrial cancer

\section{Schlüsselwörter}

- operative, medikamentöse und palliative Therapie des Zervixkarzinoms

operative, adjuvante und palliative Therapie des Endometriumkarzinoms
Bibliography

Dol http://dx.doi.org/

10.1055/s-0033-1350825

Geburtsh Frauenheilk 2013; 73 :

908-910 @ Georg Thieme

Verlag KG Stuttgart · New York . ISSN 0016-5751

\section{Correspondence}

Prof. Dr. med. Peter Mallmann

Klinik und Poliklinik für Frauen-

heilkunde und Geburtshilfe

Universitätsklinikum Köln (AöR)

Kerpener Straße 34

50931 Köln

Peter.Mallmann@uk-koeln.de

\section{Abstract}

The S2k guideline "Diagnostics and Therapy for Cervical Cancer" published in 2008 is currently being revised to the S3 level. Current developments in epidemiology, surgical therapy, radiochemotherapy and drug therapy will be presented. The S2k guideline "Diagnostics and Therapy for Endometrial Cancer" will also be up-dated this year. The revised recommendations on early diagnosis and diagnostics, therapy for precursors, surgical therapy, adjuvant therapy and therapy for recurrences and metastases will be presented.

\section{$\nabla$}

\section{Epidemiology}

In Germany each year 6500 women develop cervical cancer and 1700 die due to the disease. The age distribution of this disease shows a peak between 35 and 54 years and a second peak at 65 years of age, in the past 25 years the average age at first diagnosis has decreased by 14 years. $80 \%$ of all cervical cancers are squamous cell carcinomas; the proportion of adenocarcinomas has doubled from 10 to $20 \%$ in the last 20 years. While the incidence of invasive cervical cancer in Germany has remained more or less constant, the incidence of cervical precanceroses has increased 4fold within the past few years.

A prerequisite for the development of cervical cancer is an infection with high-risk HPV.

\section{Surgical therapy}

In principle, for all cervical cancers where a complete resection seems possible on the basis of clinical estimations, if necessary supplemented by imaging diagnostics, an operation is recommended. Radiochemotherapy in the stages IB and

\section{Zusammenfassung}

$\nabla$

Die S2k-Leitline „Diagnostik und Therapie des Zervixkarzinoms“ aus dem Jahr 2008 wird derzeit auf S3-Niveau angehoben. Die aktuellen Entwicklungen der Epidemiologie, operativen Therapie, Radiochemotherapie und medikamentösen Therapie werden dargestellt. Die S2k-Leitlinien „Diagnostik und Therapie des Endometriumkarzinoms" wurden in diesem Jahr ebenfalls aktualisiert. Die aktualisierten Empfehlungen zur Früherkennung und Diagnostik, Therapie der Vorstufen, operativen Therapie, adjuvanten Therapie und Therapie bei Rezidiven und Metastasen werden dargestellt.

II would probably give rise to similar long-term results but with differing side effect profiles, however data from prospective comparative randomised studies are not yet available.

In stage IA1 a fertility-conserving therapy in the form of a conisation, in cases with completed family planning a hysterectomy is indicated. In the presence of risk factors such as, for example, an extensive lymphangiosis, a supplementary pelvic lymphadenectomy should be performed.

In stages IA2 and IB1 fertility-conserving therapy in the form of a trachelectomy is possible, provided that no further risk factors such as L1, V1 or adenocarcinoma are present.

In stages IB2 to IIB one should start with a lymphadenectomy para-aortal-caudal to the inferior mesenteric artery. When lymph nodes have been attacked, the performance of a complete paraaortal lymphadenectomy to the renal pedicle is indicated. If the upper para-aortic lymph nodes are also positive the operation must be terminated.

An organ-conserving procedure such as a conisation or trachelectomy is contraindicated when the tumour is greater than $2 \mathrm{~cm}$ or, respectively, 
the remaining cervix $<1 \mathrm{~cm}$, in the presence of lymph node metastases, lymphangiosis or haemangiosis, G3 or special histological types such as, e.g., neuroendocrine carcinoma or adenocarcinoma. When these exclusion criteria are followed all other procedures for organ-conserving treatment of cervical cancer are equally effective.

Several different operative procedures are available for the surgical treatment of cervical cancer: the classic abdominal operation according to Wertheim-Meigs in its various modifications, the various options for laparoscopic hysterectomy, either as laparoscope-assisted vaginal or as vaginally assisted laparoscopic radical hysterectomy. The principle behind surgical therapy for cervical cancer is the radical removal of the tumour with adequate disease-free margins, the extent of radicality is determined by the spread of the tumour. A surgical alternative is the total mesometrial resection (TMMR). In this concept oncological safety is not determined by the maintenance of specific safety margins, instead complete removal of the afflicted embryonic compartment is decisive.

\section{Radiochemotherapy}

\section{Primary radiochemotherapy}

Primary radiotherapy is indicated in cases where an operation is not possible due the patient's general condition or an unacceptably high surgical risk or when the upper para-aortic lymph nodes are affected in stages IB2, IIA, IIB, and is obligatory in stage III and higher. The radiotherapy should be performed as radiochemotherapy with simultaneous administration of cisplatin at a dose of $40 \mathrm{mg} / \mathrm{m}^{2}$ body area weekly for 5 cycles.

\section{Adjuvant radiochemotherapy}

In the presence of a risk factor such as, for example, positive lymph nodes, $\mathrm{G} 3$, tumour size $>4 \mathrm{~cm}$, inadequate lymphadenectomy, R1 resection, deep stromal invasion, extensive parametrial infiltration, extensive lymphangiosis or haemangiosis, an adjuvant radiochemotherapy should be performed. Through suitable pre-therapeutic selection, if necessary with the help of imaging procedures, the patients should be subjected to only one therapeutic option, i.e., either only surgery or only radiochemotherapy. Performance of a secondary hysterectomy after primary radiochemotherapy merely increases the risk of complications without any beneficial effect on the oncological results. During radiochemotherapy, if necessary by means of blood transfusions care should be taken to maintain the Hb value above $12 \mathrm{~g} / \mathrm{dL}$. The administration of erythropoietin under on-going radiochemotherapy is contraindicated.

\section{Drug therapy}

Performance of adjuvant chemotherapy after a primary radical operation due to risk factors such as, e.g., positive lymph nodes does not, according to all currently available studies, lead to any significant beneficial effect. If the corresponding risk factors are already present at the primary diagnosis, e.g., bulky disease with a tumour diameter $>4 \mathrm{~cm}$ or an imaging diagnostic suspicion of affected lymph nodes, there is a further option to perform a neoadjuvant chemotherapy. This should consist of a platinum-containing chemotherapy performed at shortened intervals with more intensive dosing. The suitability for surgery can be improved by neoadjuvant chemotherapy since it can reduce the size of the tumour, reduce the incidence of positive lymph nodes and reduce parametrial infiltration. Data are available showing that the performance of neoadjuvant chemotherapy can possibly im- prove the progression-free survival and that, in combination with a subsequent operation or radiotherapy, may even be superior to a sole operation or a sole radiotherapy.

\section{Recurrences and metastases}

Upon suspicion of a tumour recurrence an attempt at histological conformation should be undertaken along with imaging diagnostics to assess the spread. For central recurrences after radical hysterectomy when disseminating metastases have been excluded, there is a possibility for radiochemotherapy or exenteration. For central recurrences after radiochemotherapy, exenteration is the only option provided that an R0 resection is possible. In the absence of a possibility for complete resection after radiochemotherapy the only option left is the best supportive care.

In cases of isolated distant metastases, local resectioning or destructive procedures such as, e.g., radiofrequency ablation are available. When complete resection or destruction of the metastases can be achieved, in isolated cases, an extension of the progression-free survival is possible. In the cases of disseminated metastases or those not accessible for local therapy, the only option remaining is a palliative chemotherapy with cisplatin in combination with paclitaxel or topotecan.

\section{Endometrial Cancer \\ $\nabla$}

\section{Early recognition and diagnostics}

In the numerous available studies, the performance of vaginal ultrasound in asymptomatic women has not led to a reduction in the mortality of endometrial cancer so that the installation of routine screening examinations cannot be recommended. The decisive step in the early recognition of endometrial cancer is the histological clarification of every bleeding event in the postmenopausal period as well as of any atypical bleeding in the perimenopause, irrespective of the endometrial thickness as determined by vaginal ultrasound. This histological clarification may take the form of a hysteroscopy with fractional abrasion or a non-invasive procedure to obtain biopsy specimens from the uterine body. The performance of a long-duration tamoxifen therapy is associated with a 2.7-fold higher risk for the occurrence of endometrial cancer. Here also the sensitivity and specificity of vaginal ultrasound for endometrial cancer are so low under tamoxifen that no ultrasound and a histological clarification should only be performed under this therapy regimen when bleeding disorders occur. Harvesting of specimens for histology and surgical staging cannot be replaced by any imaging procedure.

\section{Therapy for precursors of endometrial cancer}

Endometrial hyperplasias without any atypical features do not require any drug or surgical treatment. When endometrial hyperplasia with atypical features is detected, hysterectomy is indicated for both pre- and postmenopausal women on account of the high risk of degeneration. For women who still wish to have children an attempt at conservative therapy with gestagens may be undertaken. In every such case a control abrasion is necessary in order to exclude the progression of hyperplasia into an invasive cancer. An attempt at conservative treatment in these circumstances is only justified when the patient is prepared to undergo close monitoring with repeated biopsies. 


\section{Surgical therapy for endometrial cancer}

The standard therapy for endometrial cancer comprises hysterectomy and bilateral adnexectomy as well as harvesting of cytology specimens from the abdominal cavity. For low risk cases an alternative to the abdominal procedure involves a laparoscopic approach. In the presence of positive endometrial histology in stages pT1a, G1 or G2 and clinically unremarkable lymph nodes a lymphadenectomy is not necessary. From stage pT1b, each and every serous or, respectively, clear-cell cancer as well as all G3 carcinomas, pelvic and para-aortal lymphadenectomies down to the renal pedicle are obligatory. In cases with serous or clear-cell carcinomas peritoneal biopsies and an infracolic omentectomy should be done. In further advanced stages, similar to ovarian cancer, an attempt at a tumour resection as radical as possible is indicated, in order to improve the prerequisites for a postoperative systemic therapy or radiotherapy.

\section{Adjuvant therapy}

An indication for radiotherapy is given when surgical therapy cannot be performed due to pre-existing comorbidity. For patients in stage IA G3 or stage IB surgical therapy should be followed by an adjuvant brachytherapy, for those in stages IB G3, II and III this is supplemented by percutaneous irradiation. By means of adjuvant radiotherapy, at least for stages I and II, a significant reduction of the risk for local recurrences can be achieved albeit without any impact on total survival.

Patients with a low risk for local recurrences, i.e., endometrioid histology and stage IA G1-G2 are generally cured by surgery and do not need any further adjuvant therapy. Patients in stage IB G3, II and III as well as all those with serous and clear-cell endometrial carcinomas should receive adjuvant chemotherapy with platinum and paclitaxel in sequence with their radiotherapy. Adjuvant endocrine therapy with gestagens does not have any clinical benefits.

\section{Recurrences and metastases}

Upon the occurrence of local recurrences and isolated metastases with exclusion of distant metastases by imaging procedures, surgical resection should be undertaken. However, this is only meaningful when a complete resection is possible. If $\mathrm{R} 0$ resection is not possible, radiotherapy should be performed. If radiotherapy has already been carried out and an operation is not possible, systemic therapy remains as an option. For hormone receptorpositive cancers this is carried out in the form of a gestagen therapy. For patients with hormone receptor-negative tumours and with extra-abdominal distant metastases, the possibility of a palliative chemotherapy, e.g., combination chemotherapy with paclitaxel and carboplatin may be decided upon in a case by case manner.

In 2013 the S2 guideline "Endometrial Cancer" was updated, an update of the current S2 guideline "Cervical Cancer" to the S3 level is planned for 2014 .

\section{Conflict of Interest \\ No conflict of interest.}

Deutschsprachige Zusatzinformationen online abrufbar unter: www.thieme-connect.de/ejournals/toc/gebfra. 